\title{
Relativistic Toda Chain with Boundary Interaction at Root of Unity
}

\author{
Nikolai IORGOV ${ }^{\dagger}$, Vladimir ROUBTSOV ${ }^{\ddagger}$, Vitaly SHADURA ${ }^{\dagger}$ and Yuri TYKHYY ${ }^{\dagger}$ \\ $\dagger$ Bogolyubov Institute for Theoretical Physics, 14 b Metrolohichna Str., Kyiv, 03143 Ukraine \\ E-mail: iorgov@bitp.kiev.ua,shadura@bitp.kiev.ua,tykhyy@bitp.kiev.ua \\ ‡LAREMA, Dépt. de Math. Université d'Angers, 2 bd. Lavoisier, 49045, Angers, France \\ E-mail: volodya@tonton.univ-angers.fr \\ $\S$ ITEP, Moscow, 25 B. Cheremushkinskaja, 117259, Moscow, Russia
}

Received November 15, 2006, in final form January 03, 2007; Published online January 19, 2007

Original article is available at http://www.emis.de/journals/SIGMA/2007/013/

\begin{abstract}
We apply the Separation of Variables method to obtain eigenvectors of commuting Hamiltonians in the quantum relativistic Toda chain at a root of unity with boundary interaction.

Key words: quantum integrable model with boundary interaction; quantum relativistic Toda chain

2000 Mathematics Subject Classification: 81R12; 81R50
\end{abstract}

To the memory of Vadim Kuznetsov

\section{Introduction}

During the last decade, a considerable progress in application of the Separation of Variables method to a broad class of quantum integrable models has been achieved. This progress was initiated by the paper [1] of Sklyanin who has proposed (using the $R$-matrix formalism) a recipe for a Separation of Variables in the case of quantum Toda chain where the algebraic Bethe ansatz fails. The next important step was done by Kharchev and Lebedev [2] who realized an iterative procedure of obtaining the eigenfunctions of the $n$-particle open Toda chain by some integral transformation from the eigenfunctions of the $(n-1)$-particle open Toda chain. This iterative method was applied later to a relativistic Toda chain [3].

The Separation of Variables was found to be effective for the derivation of the eigenfunctions of the commuting Hamiltonians of the $n$-particle quantum Toda chain with boundary interaction $[4,5]$ in the framework of Sklyanin's approach to the integrable boundary problems [6]. In the paper [5] the $n$-particle eigenfunctions of the quantum Toda chain when the first and last particles are exponentially interacting with the walls (the two-boundary interaction) is constructed by means of an integral transformation of the eigenfunctions for the Toda chain with one-boundary interaction (the auxiliary problem). These eigenfunctions, in turn, are constructed using the eigenfunctions of the $n$-particle open Toda chain.

Recently, a Separation of Variables was applied to the $N$-state spin lattice model - the Baxter-Bazhanov-Stroganov (BBS) model $[7,8]$. In the $R$-matrix formulation of this model the cyclic $L$-operator $[9,10]$ emerged. These operators are intertwined by the six-vertex $R$-matrix at a root of unity. The cyclic $L$-operator of the BBS model reduces (at special values of parameters) to the $L$-operator of the quantum Relativistic Toda Chain (RTC) at a root of unity [11].

${ }^{\star}$ This paper is a contribution to the Vadim Kuznetsov Memorial Issue "Integrable Systems and Related Topics". The full collection is available at http://www.emis.de/journals/SIGMA/kuznetsov.html 
The goal of this contribution is to find common eigenvectors of commuting Hamiltonians of the RTC at a root of unity with boundary interaction. We use for this the approach from [5].

Let us describe briefly the structure of the paper. We start with a short account of the model under consideration and remind its properties based on the presentation in [11] (Section 2). We recall the standard Sklyanin formalism introducing a chain of $L$-operators satisfying some $R L L=$ $L L R$ relations as well as its "twisted" version (2.3) such that the corresponding ("twisted") monodromy matrix satisfies the "Reflection Equations" and some "unitarity" condition. We specify a "boundary matrix" solution $K_{+}(\lambda)$ of the dual Reflection Equations which are necessary for a description of a two-boundary RTC. We close this section with a Hamiltonian generating function to the two-boundary RTC.

We give the explicit formulas for the eigenvectors of the open RTC in the Section 3 using the eigenvectors formulas for the open Bazhanov-Stroganov quantum chain of which the open RTC is a (specified) particular case [9].

In Section 4 we consider the eigenvectors of the one-boundary RTC specifying some particular choice of the boundary matrices. We identify the coefficients of the corresponding generating function with the commuting one-boundary RTC Hamiltonians and compute the common eigenvectors. The same questions are treated in Section 5 for the two-boundary RTC using the Separation of Variables method. We write the Baxter equation for separated variables explicitly (5.3).

Section 6 contains an another proof of the commutativity of the one-boundary RTC Hamiltonians of the Section 4. We show in fact that they belong to a wide class of Hamiltonians which can be expressed like a sort of Gelfand-Retakh quasideterminants. This quasideterminants are (in our case) just Plücker coordinates $\Delta_{s} \Delta_{0}^{-1}$ on $G r_{n-1}(n)$. The commutation (and, hence the existence of the common spectrum) is guaranteed by the results of [12] (see also [13, 14]).

We finish with a short discussion in the Section 7 and we leave some tedious computations to the Appendix A.

We are dedicating this paper to the memory of Vadim Kuznetsov. His important contributions to the subject of Separation of Variables and in particularly to its applications in quantum integrable models with boundary interaction [4, 15, 16, 17] are widely acknowledged in the Mathematical Physics community. In particular, it was Kuznetsov's paper [16] where the Separation of Variables was performed for the quantum relativistic Toda chain and commuting Hamiltonians of the quantum RTC with integrable boundary interaction (the quantum RTC for general classical root systems) were obtained.

\section{The formulation of the model}

In this section we recall briefly the subject of the model called the quantum Relativistic Toda Chain (RTC) chain at root of unity [11].

Let $\omega=e^{2 \pi \mathrm{i} / N}, N \geq 2$. For each particle $k, k=1,2, \ldots, n$, of the $n$-particle RTC there is a corresponding $N$-dimensional linear space (quantum space) $\mathcal{V}_{k}$ with the basis $|\gamma\rangle_{k}, \gamma \in \mathbb{Z}_{N}$, and a pair of operators $\left\{\boldsymbol{u}_{k}, \boldsymbol{v}_{k}\right\}$ acting on $\mathcal{V}_{k}$ by the formulas:

$$
\boldsymbol{v}_{k}|\gamma\rangle_{k}=v \omega^{\gamma}|\gamma\rangle_{k}, \quad \boldsymbol{u}_{k}|\gamma\rangle_{k}=u|\gamma-1\rangle_{k}
$$

The space of quantum states of RTC with $n$ particles is $\mathcal{V}=\mathcal{V}_{1} \otimes \mathcal{V}_{2} \otimes \cdots \otimes \mathcal{V}_{n}$. We extend the action of operators $\left\{\boldsymbol{u}_{k}, \boldsymbol{v}_{k}\right\}$ to $\mathcal{V}$ defining this action to be identical on $\mathcal{V}_{s}, s \neq k$. Thus we have the following commutation relations

$$
\boldsymbol{u}_{j} \boldsymbol{u}_{k}=\boldsymbol{u}_{k} \boldsymbol{u}_{j}, \quad \boldsymbol{v}_{j} \boldsymbol{v}_{k}=\boldsymbol{v}_{k} \boldsymbol{v}_{j}, \quad \boldsymbol{u}_{j} \boldsymbol{v}_{k}=\omega^{\delta_{j, k}} \boldsymbol{v}_{k} \boldsymbol{u}_{j}
$$


Monodromy matrix for RTC with $n$ particles is

$$
T_{n}(\lambda)=L_{1}(\lambda) L_{2}(\lambda) \cdots L_{n}(\lambda)=\left(\begin{array}{cc}
A_{n}(\lambda) & B_{n}(\lambda) \\
C_{n}(\lambda) & D_{n}(\lambda)
\end{array}\right)
$$

where

$$
L_{k}(\lambda)=\left(\begin{array}{cc}
1+\frac{\varkappa}{\lambda} \boldsymbol{v}_{k} & -\frac{1}{\lambda} \boldsymbol{u}_{k} \\
\boldsymbol{u}_{k}^{-1} \boldsymbol{v}_{k} & 0
\end{array}\right), \quad k=1,2, \ldots, n .
$$

Thus the model depends on three parameters $u, v$ and $\varkappa$. It is possible to make this model to be inhomogeneous by attaching the index $k$ to the parameters $u, v$ and $\varkappa$. All the results presented in this paper can be directly generalized to inhomogeneous case.

The straightforward calculation shows that the operators $L_{k}(\lambda)$ satisfy the following commutation relations:

$$
R(\lambda, \mu) L_{k}^{(1)}(\lambda) L_{k}^{(2)}(\mu)=L_{k}^{(2)}(\mu) L_{k}^{(1)}(\lambda) R(\lambda, \mu),
$$

where the standard notations $L_{k}^{(1)}(\lambda)=L_{k}(\lambda) \otimes \mathbb{I}, L_{k}^{(2)}(\mu)=\mathbb{I} \otimes L_{k}(\mu)$ were used and the $R$-matrix $R(\lambda, \mu)$ has the form

$$
R(\lambda, \mu)=\left(\begin{array}{cccc}
\lambda-\omega \mu & 0 & 0 & 0 \\
0 & \lambda-\mu & (1-\omega) \mu & 0 \\
0 & (1-\omega) \lambda & \omega(\lambda-\mu) & 0 \\
0 & 0 & 0 & \lambda-\omega \mu
\end{array}\right) .
$$

Using (2.2), we obtain the following relation for monodromy matrix

$$
R(\lambda, \mu) T_{n}^{(1)}(\lambda) T_{n}^{(2)}(\mu)=T_{n}^{(2)}(\mu) T_{n}^{(1)}(\lambda) R(\lambda, \mu) .
$$

We will use the method [6] of Sklyanin for description of RTC with integrable boundary interaction. The $L$-operator (2.1) together with

$$
\widetilde{L}_{k}(\lambda)=\lambda \sigma_{x} L_{k}^{-1}(\theta / \lambda) \sigma_{x}=\left(\begin{array}{cc}
\varkappa \lambda+\omega \theta \boldsymbol{v}_{k}^{-1} & -\theta \boldsymbol{u}_{k}^{-1} \\
\lambda \boldsymbol{v}_{k}^{-1} \boldsymbol{u}_{k} & 0
\end{array}\right)=\omega \theta \boldsymbol{v}_{k}^{-1} \sigma_{z} L_{k}(\omega \theta / \lambda)^{T} \sigma_{z}
$$

satisfy the following relations

$$
\begin{aligned}
& R(\lambda, \mu) L_{k}^{(1)}(\lambda) L_{k}^{(2)}(\mu)=L_{k}^{(2)}(\mu) L_{k}^{(1)}(\lambda) R(\lambda, \mu), \\
& R(\lambda, \mu) \widetilde{L}_{k}^{(1)}(\lambda) \widetilde{L}_{k}^{(2)}(\mu)=\widetilde{L}_{k}^{(2)}(\mu) \widetilde{L}_{k}^{(1)}(\lambda) R(\lambda, \mu), \\
& \widetilde{L}_{k}^{(2)}(\mu) \widetilde{R}(\lambda, \mu) L_{k}^{(1)}(\lambda)=L_{k}^{(1)}(\lambda) \widetilde{R}(\lambda, \mu) \widetilde{L}_{k}^{(2)}(\mu), \\
& L_{k}^{(2)}(\mu) \widetilde{R}(\lambda, \mu) \widetilde{L}_{k}^{(1)}(\lambda)=\widetilde{L}_{k}^{(1)}(\lambda) \widetilde{R}(\lambda, \mu) L_{k}^{(2)}(\mu),
\end{aligned}
$$

where

$$
\begin{aligned}
& \widetilde{R}(\lambda, \mu)=\mu \sigma_{x}^{(2)} R(\lambda, \theta / \mu) \sigma_{x}^{(2)}=\left(\begin{array}{llll}
\theta-\lambda \mu & 0 & 0 & \omega \theta-\theta \\
0 & \omega \theta-\lambda \mu & 0 & 0 \\
0 & 0 & \omega \theta-\lambda \mu & 0 \\
\omega \lambda \mu-\lambda \mu & 0 & 0 & \omega \theta-\omega \lambda \mu
\end{array}\right), \\
& \sigma_{x}=\left(\begin{array}{ll}
0 & 1 \\
1 & 0
\end{array}\right), \quad \sigma_{z}=\left(\begin{array}{ll}
1 & 0 \\
0 & -1
\end{array}\right) .
\end{aligned}
$$


Here $\theta$ is an arbitrary parameter. It is important that $\widetilde{R}(\mu, \lambda)=\widetilde{R}(\lambda, \mu)=P_{12} \widetilde{R}(\lambda, \mu) P_{12}$, where the operator $P_{12}$ is the operator of permutation of two spaces. Then the matrix

$$
U(\lambda)=\widetilde{L}_{n}(\lambda) \cdots \widetilde{L}_{1}(\lambda) K_{-}(\lambda) L_{1}(\lambda) \cdots L_{n}(\lambda)=\left(\begin{array}{cc}
\mathcal{A}(\lambda) & \mathcal{B}(\lambda) \\
\mathcal{C}(\lambda) & \mathcal{D}(\lambda)
\end{array}\right)
$$

satisfies the reflection equation

$$
R(\lambda, \mu) U^{(1)}(\lambda) \widetilde{R}(\lambda, \mu) U^{(2)}(\mu)=U^{(2)}(\mu) \widetilde{R}(\lambda, \mu) U^{(1)}(\lambda) R(\lambda, \mu)
$$

if $K_{-}(\lambda)$ satisfies the same equation:

$$
R(\lambda, \mu) K_{-}^{(1)}(\lambda) \widetilde{R}(\lambda, \mu) K_{-}^{(2)}(\mu)=K_{-}^{(2)}(\mu) \widetilde{R}(\lambda, \mu) K_{-}^{(1)}(\lambda) R(\lambda, \mu)
$$

The solution $K_{-}(\lambda)$ with non-operator matrix elements is

$$
K_{-}(\lambda)=\left(\begin{array}{ll}
\alpha_{-}\left(\frac{\theta}{\lambda}-\lambda\right) & \eta_{-}+\beta_{-} \frac{\theta}{\lambda} \\
\eta_{-}+\beta_{-} \lambda & \delta_{-}\left(\frac{\theta}{\lambda}-\lambda\right)
\end{array}\right) .
$$

This solution depends on four parameters $\alpha_{-}, \beta_{-}, \delta_{-}, \eta_{-}$. It can be found directly solving (2.5) or adapting the formulas from [18] (see also [16]). The matrix $U(\lambda)$ satisfies the so-called unitarity condition:

$$
\begin{aligned}
& U(\omega \theta / \lambda)=\left(\begin{array}{ll}
\mathcal{A}(\omega \theta / \lambda) & \mathcal{B}(\omega \theta / \lambda) \\
\mathcal{C}(\omega \theta / \lambda) & \mathcal{D}(\omega \theta / \lambda)
\end{array}\right) \\
& =\frac{1}{\omega\left(\theta-\lambda^{2}\right)}\left(\begin{array}{ll}
\left(\lambda^{2}-\omega^{2} \theta\right) \mathcal{A}(\lambda) & \lambda^{2}(1-\omega) \mathcal{B}(\lambda)+\left(\omega \theta-\lambda^{2}\right) \mathcal{C}(\lambda) \\
\omega\left(\omega \theta-\lambda^{2}\right) \mathcal{B}(\lambda)+\omega \theta(1-\omega) \mathcal{C}(\lambda) & \left(\lambda^{2}-\omega^{2} \theta\right) \mathcal{D}(\lambda)
\end{array}\right) .
\end{aligned}
$$

We have $\mathcal{A}(\lambda) \mathcal{A}(\mu)=\mathcal{A}(\mu) \mathcal{A}(\lambda)$ from the reflection equation (2.4). It means that $\mathcal{A}(\lambda)$ is generation function with respect to $\lambda$ for commuting set of operators. These operators are Hamiltonians for one-boundary RTC.

To describe a two-boundary RTC we need the dual reflection equation:

$$
R(\lambda, \mu) K_{+}^{(2)}(\mu) Q(\lambda, \mu) K_{+}^{(1)}(\lambda)=K_{+}^{(1)}(\lambda) Q(\lambda, \mu) K_{+}^{(2)}(\mu) R(\lambda, \mu)
$$

where

$$
Q(\lambda, \mu)=\left(\left(\widetilde{R}^{t_{1}}(\lambda, \mu)\right)^{-1}\right)^{t_{1}}
$$

Its solution is

$$
K_{+}(\lambda)=\left(\begin{array}{ll}
\alpha_{+}\left(\frac{\omega^{2} \theta}{\lambda}-\lambda\right) & \eta_{+}+\beta_{+} \frac{\omega \theta}{\lambda} \\
\eta_{+} \omega+\beta_{+} \lambda & \delta_{+}\left(\frac{\omega \theta}{\lambda}-\omega^{-1} \lambda\right)
\end{array}\right) .
$$

Then the generation function for the Hamiltonians of the two-boundary RTC is given by $\boldsymbol{t}(\lambda)=$ $\operatorname{Tr}\left(K_{+}(\lambda) U(\lambda)\right)$ or explicitly:

$$
\begin{aligned}
\boldsymbol{t}(\lambda)= & \alpha_{+}\left(\theta \omega^{2} / \lambda-\lambda\right) \mathcal{A}(\lambda)+\left(\beta_{+} \lambda+\eta_{+} \omega\right) \mathcal{B}(\lambda) \\
& +\left(\beta_{+} \theta \omega / \lambda+\eta_{+}\right) \mathcal{C}(\lambda)+\delta_{+}\left(\omega \theta / \lambda-\omega^{-1} \lambda\right) \mathcal{D}(\lambda) .
\end{aligned}
$$




\section{Eigenvectors for the open RTC}

In order to give explicit formulas for the eigenvectors for RTC we remind the definition (see for example [19]) of $w_{p}(s)$ which is an analogue of $\Gamma_{q}$-function at root of unity. For any point $p=(x, y)$ of Fermat curve $x^{N}+y^{N}=1$, we define $w_{p}(s), s \in \mathbb{Z}_{N}$, by

$$
\frac{w_{p}(s)}{w_{p}(s-1)}=\frac{y}{1-x \omega^{s}}, \quad w_{p}(0)=1
$$

Due to the Fermat curve condition the function $w_{p}(s)$ is cyclic: $w_{p}(s+N)=w_{p}(s)$.

The explicit formulas for the eigenvectors of open RTC, that is eigenvectors of $A_{n}(\lambda)$, can be extracted from [8], where the formulas for the eigenvectors of open Bazhanov-Stroganov quantum chain (which includes RTC) were obtained. Here we give the resulting formulas specialized to open RTC with a little change of notations.

The eigenvectors of open RTC are given in terms of $w_{p}(s)$ defined by the different points $p_{m, s ; m^{\prime}, s^{\prime}}=\left(x_{m, s ; m^{\prime}, s^{\prime}}, y_{m, s ; m^{\prime}, s^{\prime}}\right), m, m^{\prime}=1,2, \ldots, n ; s=1,2, \ldots, m ; s^{\prime}=1,2, \ldots, m^{\prime}$, belonging to Fermat curve $x^{N}+y^{N}=1$. The $x$-coordinates of these points are defined by unknown parameters (amplitudes) $v_{m, s}$ by $x_{m, s ; m^{\prime}, s^{\prime}}=v_{m, s} / v_{m^{\prime}, s^{\prime}}$. Then the corresponding $y_{m, s ; m^{\prime}, s^{\prime}}$ are defined up to a root of 1 . The parameters $v_{m, s}$ are defined by the initial value $v_{11}:=v$ and the recurrent relations

$$
\begin{aligned}
& v_{m 1} v_{m 2} \cdots v_{m m}=v_{m-1,1} v_{m-1,2} \cdots v_{m-1, m-1} v, \\
& \varkappa \prod_{s \neq l} \frac{y_{m-1, s ; m-1, l}}{y_{m-1, l ; m-1, s}} \frac{\prod_{k=1}^{m} y_{m-1, l ; m, k}}{\prod_{s=1}^{m-2} y_{m-2, s ; m-1, l}}=1, \quad l=1,2, \ldots, m-1 .
\end{aligned}
$$

In the case of homogeneous RTC these relations can be solved explicitly [11] (see also [8]).

We will use the notation $\left|\gamma_{n}\right\rangle \in \mathcal{V}_{1} \otimes \cdots \otimes \mathcal{V}_{n}$ for the eigenvectors of the operator $A_{n}(\lambda)$ of the open RTC with $n$ particles. These eigenvectors are labelled by $n$ parameters $\gamma_{n, s} \in \mathbb{Z}_{N}$, $s=1,2, \ldots, n$, collected into a vector

$$
\gamma_{n}=\left(\gamma_{n, 1}, \ldots, \gamma_{n, n}\right) \in\left(\mathbb{Z}_{N}\right)^{n}
$$

The formula

$$
\left|\gamma_{n}\right\rangle=\sum_{\gamma_{n-1}} \mu\left(\gamma_{n-1}\right) Q\left(\gamma_{n-1} \mid \gamma_{n}\right)\left|\gamma_{n-1}\right\rangle \otimes\left|\sum_{k=1}^{n} \gamma_{n, k}-\sum_{l=1}^{n-1} \gamma_{n-1, l}\right\rangle_{n}
$$

where

$$
\begin{aligned}
& \mu\left(\gamma_{n-1}\right)=\prod_{\substack{j, l=1 \\
(j \neq l)}}^{n-1} w_{p_{n-1, j ; n-1, l}}\left(\gamma_{n-1, j}-\gamma_{n-1, l}-1\right), \\
& Q\left(\gamma_{n-1} \mid \gamma_{n}\right)=\frac{\omega^{\gamma_{n-1,1}+\cdots+\gamma_{n-1, n-1}}}{\prod_{k=1}^{n} \prod_{l=1}^{n-1} w_{p_{n-1, l ; n, k}}\left(\gamma_{n-1, l}-\gamma_{n, k}-1\right)},
\end{aligned}
$$

gives the eigenvectors $\left|\gamma_{n}\right\rangle$ of $A_{n}(\lambda)$ from the eigenvectors $\left|\gamma_{n-1}\right\rangle \in \mathcal{V}_{1} \otimes \cdots \otimes \mathcal{V}_{n-1}$ of $A_{n-1}(\lambda)$ and basis vectors $|\gamma\rangle_{n} \in \mathcal{V}_{n}$. To find the formula for $\left|\gamma_{n-1}\right\rangle$ we can use the same formula and 
so on. At the last step we need the eigenvectors of 1-particle RTC which are just basis vectors $\left|\gamma_{1,1}\right\rangle_{1} \in \mathcal{V}_{1}$. The vectors $\left|\gamma_{n}\right\rangle$ obtained by the described procedure satisfy

$$
\begin{aligned}
& A_{n}(\lambda)\left|\gamma_{n}\right\rangle=\prod_{k=1}^{n}\left(1+\frac{\varkappa v_{n, k} \omega^{\gamma_{n, k}}}{\lambda}\right)\left|\gamma_{n}\right\rangle=\prod_{k=1}^{n}\left(1-\frac{\lambda_{n, k}}{\lambda}\right)\left|\gamma_{n}\right\rangle, \\
& B_{n}\left(\lambda_{n, k}\right)\left|\gamma_{n}\right\rangle=-\frac{h_{k}}{\lambda_{n, k}}\left|\gamma_{n}^{-k}\right\rangle, \\
& B_{n}(\lambda)\left|\gamma_{n}\right\rangle=-\frac{h_{k}}{\lambda} \sum_{k=1}^{n} \prod_{s \neq k}\left(\frac{\lambda_{n, k}\left(\lambda-\lambda_{n, s}\right)}{\lambda\left(\lambda_{n, k}-\lambda_{n, s}\right)}\right)\left|\gamma_{n}^{-k}\right\rangle,
\end{aligned}
$$

where $\lambda_{n, k}=-\varkappa v_{n, k} \omega^{\gamma_{n, k}}$ and $h_{k}=u \prod_{l=1}^{n-1} y_{n-1, l ; n, k}$. In the above formulas the vector $\left|\gamma_{n}^{-k}\right\rangle$ means the vector $\left|\gamma_{n 1}, \ldots, \gamma_{n n}\right\rangle$ with $\gamma_{n, k}$ replaced by $\gamma_{n, k}-1$.

We will omit in what follows the index $n$ in matrix elements of monodromy matrix.

At $\lambda=\omega \mu$, the rank of $R(\lambda, \mu)$ becomes 1 . Therefore both sides of (2.2) become proportional to $R(\lambda, \mu)$ with the coefficient being the so-called quantum determinant:

$$
\text { qdet } T_{n}(\mu)=D(\omega \mu) A(\mu)-B(\omega \mu) C(\mu)=\operatorname{qdet} L_{1}(\mu) \cdots \operatorname{qdet} L_{n}(\mu)=\frac{\mathbf{V}}{(\omega \mu)^{n}},
$$

where

$$
\mathbf{V}=\prod_{k=1}^{n} \boldsymbol{v}_{k}, \quad \mathbf{V}\left|\boldsymbol{\gamma}_{n}\right\rangle=\prod_{k=1}^{n}\left(v_{n, k} \omega^{\gamma_{n, k}}\right)\left|\boldsymbol{\gamma}_{n}\right\rangle .
$$

Now use (3.2) for $\mu=\lambda_{n, r}$ :

$$
D\left(\omega \lambda_{n, r}\right) A\left(\lambda_{n, r}\right)-B\left(\omega \lambda_{n, r}\right) C\left(\lambda_{n, r}\right)=\frac{\mathbf{V}}{\left(\omega \lambda_{n, r}\right)^{n}} .
$$

Acting by both sides on $\left|\gamma_{n}\right\rangle$ we get

$$
-B\left(\omega \lambda_{n, r}\right) C\left(\lambda_{n, r}\right)\left|\gamma_{n}\right\rangle=\frac{\prod_{k=1}^{n}\left(v_{n, k} \omega^{\gamma_{n, k}}\right)}{\left(\omega \lambda_{n, r}\right)^{n}}\left|\gamma_{n}\right\rangle .
$$

Therefore

$$
C\left(\lambda_{n, r}\right)\left|\gamma_{n}\right\rangle=\frac{\prod_{k=1}^{n}\left(v_{n, k} \omega^{\gamma_{n, k}}\right)}{\left(\omega \lambda_{n, r}\right)^{n-1} h_{r}}\left|\gamma_{n}^{+r}\right\rangle .
$$

Since $C(\lambda)$ is a polynomial in $1 / \lambda$ of degree $n-1$ we can reconstruct the action of $C(\lambda)$ :

$$
C(\lambda)\left|\gamma_{n}\right\rangle=\sum_{r=1}^{n}\left(\prod_{m \neq r} \frac{\lambda_{n, r}\left(\lambda-\lambda_{n, m}\right)}{\lambda\left(\lambda_{n, r}-\lambda_{n, m}\right)}\right) C\left(\lambda_{n, r}\right)\left|\gamma_{n}\right\rangle .
$$

Finally using (3.2) after some calculation we get

$$
\begin{aligned}
D(\lambda)\left|\gamma_{n}\right\rangle= & \frac{\omega \varkappa}{\lambda} \sum_{r=1}^{n} \sum_{k \neq r}\left[\frac{\prod_{m} v_{n, m} \omega^{\gamma_{n, m}}}{(-\varkappa \omega)^{n}}\left(\frac{v_{n, k} \omega^{\gamma_{n, k}}}{\left(v_{n, k} \omega^{\gamma_{n, k}}-\omega v_{n, r} \omega^{\gamma_{n, r}}\right)\left(v_{n, r} \omega^{\gamma_{n, r}}-v_{n, k} \omega^{\gamma_{n, k}}\right)}\right)\right. \\
& \left.\times \prod_{s \neq k, r}\left(\frac{v_{n, k} \omega^{\gamma_{n, k}}\left(\lambda+\varkappa v_{n, s} \omega^{\gamma_{n, s}}\right)}{\lambda\left(v_{n, k} \omega^{\gamma_{n, k}}-v_{n, s} \omega^{\gamma_{n, s}}\right)\left(v_{n, r} \omega^{\gamma_{n, r}}-v_{n, s} \omega^{\left.\gamma_{n, s}\right)}\right.}\right)\left(\frac{h_{k}}{h_{r}}\left|\gamma_{n}^{+r-k}\right\rangle-\left|\gamma_{n}\right\rangle\right)\right] .
\end{aligned}
$$




\section{Eigenvectors for the one-boundary RTC}

We restrict our attention in what follows to the choice $\beta_{ \pm}=\delta_{ \pm}=0$. In this case the boundary matrices become

$$
K_{-}(\lambda)=\left(\begin{array}{ll}
\alpha_{-}\left(\frac{\theta}{\lambda}-\lambda\right) & \eta_{-} \\
\eta_{-} & 0
\end{array}\right), \quad K_{+}(\lambda)=\left(\begin{array}{ll}
\alpha_{+}\left(\frac{\omega^{2} \theta}{\lambda}-\lambda\right) & \eta_{+} \\
\eta_{+} \omega & 0
\end{array}\right) .
$$

Due to (2.3) we have

$$
\widetilde{L}_{n}(\lambda) \cdots \widetilde{L}_{1}(\lambda)=(\omega \theta)^{n} \mathbf{V}^{-1} \sigma_{z} T^{t}(\omega \theta / \lambda) \sigma_{z}=(\omega \theta)^{n} \mathbf{V}^{-1}\left(\begin{array}{ll}
A(\omega \theta / \lambda) & -C(\omega \theta / \lambda) \\
-B(\omega \theta / \lambda) & D(\omega \theta / \lambda)
\end{array}\right) .
$$

Therefore

$$
\begin{aligned}
& \mathcal{A}(\lambda)=(\omega \theta)^{n} \mathbf{V}^{-1}\left[\alpha_{-} \frac{\theta-\lambda^{2}}{\lambda} A(\omega \theta / \lambda) A(\lambda)+\eta_{-}(A(\omega \theta / \lambda) C(\lambda)-C(\omega \theta / \lambda) A(\lambda))\right], \\
& \mathcal{B}(\lambda)=(\omega \theta)^{n} \mathbf{V}^{-1}\left[\alpha_{-} \frac{\theta-\lambda^{2}}{\lambda} A(\omega \theta / \lambda) B(\lambda)+\eta_{-}(A(\omega \theta / \lambda) D(\lambda)-C(\omega \theta / \lambda) B(\lambda))\right], \\
& \mathcal{C}(\lambda)=(\omega \theta)^{n} \mathbf{V}^{-1}\left[-\alpha_{-} \frac{\theta-\lambda^{2}}{\lambda} B(\omega \theta / \lambda) A(\lambda)+\eta_{-}(-B(\omega \theta / \lambda) C(\lambda)+D(\omega \theta / \lambda) A(\lambda))\right], \\
& \mathcal{D}(\lambda)=(\omega \theta)^{n} \mathbf{V}^{-1}\left[-\alpha_{-} \frac{\theta-\lambda^{2}}{\lambda} B(\omega \theta / \lambda) B(\lambda)+\eta_{-}(-B(\omega \theta / \lambda) D(\lambda)+D(\omega \theta / \lambda) B(\lambda))\right] .
\end{aligned}
$$

From (2.6) it follows that $\widetilde{\mathcal{A}}(\lambda)$ defined by $\widetilde{\mathcal{A}}(\lambda)=\lambda \mathcal{A}(\lambda) /\left(\theta-\lambda^{2}\right)$, or explicitly

$$
\widetilde{\mathcal{A}}(\lambda)=(\omega \theta)^{n} \mathbf{V}^{-1}\left[\alpha_{-} A(\omega \theta / \lambda) A(\lambda)+\frac{\eta_{-} \lambda}{\left(\theta-\lambda^{2}\right)}(A(\omega \theta / \lambda) C(\lambda)-C(\omega \theta / \lambda) A(\lambda))\right],
$$

satisfies $\widetilde{\mathcal{A}}\left(\frac{\omega \theta}{\lambda}\right)=\widetilde{\mathcal{A}}(\lambda)$. It means that $\widetilde{\mathcal{A}}(\lambda)$ can be presented as

$$
\widetilde{\mathcal{A}}(\lambda)=\alpha_{-} \varkappa^{n}\left((\lambda+\omega \theta / \lambda)^{n}+(\lambda+\omega \theta / \lambda)^{n-1} H_{1}^{\mathrm{B}}+\cdots+(\lambda+\omega \theta / \lambda) H_{n-1}^{\mathrm{B}}+H_{n}^{\mathrm{B}}\right),
$$

where the set of operators $H_{1}^{\mathrm{B}}, H_{2}^{\mathrm{B}}, \ldots, H_{n}^{\mathrm{B}}$ have to be identified with the commuting set of Hamiltonians for the one-boundary RTC. Explicitly, $H_{1}^{\mathrm{B}}$ is given by $(5.2)$ with $\eta_{+}=0$. Our problem is to find the common eigenvectors for this set.

For $\boldsymbol{\sigma}=\left(\sigma_{1}, \ldots, \sigma_{n}\right) \in\left(\mathbb{Z}_{\mathbb{N}}\right)^{n}$, let

$$
\Psi_{\sigma}=\sum_{\gamma_{n}} Q\left(\gamma_{n}, \boldsymbol{\sigma}\right)\left|\gamma_{n}\right\rangle
$$

where the sum includes all $N^{n}$ combinations of $\gamma_{n}=\left\{\gamma_{n, 1}, \ldots, \gamma_{n, n}\right\}$ and

$$
\begin{aligned}
Q\left(\gamma_{n}, \boldsymbol{\sigma}\right)= & \prod_{r=1}^{n} \omega^{\gamma_{n, r}^{2}+\gamma_{n, r}} \prod_{k=1}^{n} \prod_{r=1}^{n}\left(w_{p_{r, k}^{\mathrm{B}}}\left(\gamma_{n, r}-\sigma_{k}\right) w_{\tilde{p}_{r, k}^{\mathrm{B}}}\left(\gamma_{n, r}+\sigma_{k}\right)\right) \\
& \times \prod_{r<r^{\prime}}\left(\frac{v_{n, r} \omega^{\gamma_{n, r}}-v_{n, r^{\prime}} \omega^{\gamma_{n, r^{\prime}}}}{w_{\tilde{r}_{r, r^{\prime}}}\left(\gamma_{n, r}+\gamma_{n, r^{\prime}}\right)}\right) .
\end{aligned}
$$

Then, as it will be shown in the Appendix A, we have

$$
\widetilde{\mathcal{A}}(\lambda) \Psi_{\boldsymbol{\sigma}}=\alpha_{-} \prod_{k=1}^{n}\left(\frac{\left(\lambda+\varkappa s_{k} \omega^{\sigma_{k}}\right)\left(\omega \theta / \lambda+\varkappa s_{k} \omega^{\sigma_{k}}\right)}{s_{k} \omega^{\sigma_{k}}}\right) \Psi_{\boldsymbol{\sigma}}
$$




$$
=\alpha_{-}(-\varkappa)^{n} \prod_{k=1}^{n}\left(\frac{\left(\lambda-\lambda_{k}\right)\left(\omega \theta / \lambda-\lambda_{k}\right)}{\lambda_{k}}\right) \Psi_{\boldsymbol{\sigma}}
$$

where $s_{k}, k=1,2, \ldots, n$, are some fixed amplitudes which will be defined later. Also we have used a short notation $\lambda_{k}=-\varkappa s_{k} \omega^{\sigma_{k}}$. This form of the eigenvalue polynomial corresponds to (4.3). We are using three types of points $\tilde{p}_{r, r^{\prime}}, p_{r, k}^{\mathrm{B}}$ and $\tilde{p}_{r, k}^{\mathrm{B}}$ belonging to the Fermat curve $x^{N}+y^{N}=1$ in the definition of $Q\left(\boldsymbol{\gamma}_{n}, \boldsymbol{\sigma}\right)$. They are defined by their $x$-coordinates:

$$
\tilde{x}_{r, r^{\prime}}=\varkappa^{2} v_{n, r} v_{n, r^{\prime}} /(\omega \theta), \quad x_{r, k}^{\mathrm{B}}=v_{n, r} / s_{k}, \quad \tilde{x}_{r, k}^{\mathrm{B}}=\varkappa^{2} v_{n, r} s_{k} /(\omega \theta)
$$

and the equations

$$
\frac{\eta_{-} v_{n, r}^{2}}{\varkappa^{n-2} \omega^{n+1} \theta} \prod_{r^{\prime} \neq r} \tilde{y}_{r, r^{\prime}}=\alpha_{-} h_{r} \prod_{k=1}^{n} y_{r, k}^{\mathrm{B}} \tilde{y}_{r, k}^{\mathrm{B}}, \quad r=1,2, \ldots, n .
$$

To solve these equations we have to take the $N$-th degree of both parts of the equations (4.7) and solve them with respect to elementary symmetric polynomials of $s_{k}^{N}, k=1,2, \ldots, n$. This information is enough to find the values of $s_{k}$ and the coordinates of the Fermat points entering $Q\left(\gamma_{n}, \boldsymbol{\sigma}\right)$.

Taking into account Laurent polynomial structure of $\mathcal{C}(\lambda)$ in $\lambda$ it is clear that it is enough to know the action formulas for $\mathcal{C}(\lambda)$ on $\Psi_{\boldsymbol{\sigma}}$ in $2 n+2$ points of $\lambda$ to reconstruct $\mathcal{C}(\lambda) \Psi_{\boldsymbol{\sigma}}$. The following formulas are valid:

$$
\begin{aligned}
& \mathcal{C}\left(\lambda_{k}\right) \Psi_{\boldsymbol{\sigma}}=\eta_{-} f_{k} \Psi_{\boldsymbol{\sigma}^{+k}}, \quad \mathcal{C}\left(\omega \theta / \lambda_{k}\right) \Psi_{\boldsymbol{\sigma}}=\eta_{-}(\omega \theta)^{n} f_{k}^{-1} \Psi_{\boldsymbol{\sigma}^{-k}} \\
& \mathcal{C}( \pm \sqrt{\theta}) \Psi_{\boldsymbol{\sigma}}=\eta_{-}( \pm \sqrt{\theta})^{n} \Psi_{\boldsymbol{\sigma}}
\end{aligned}
$$

where $\lambda_{k}=-\varkappa s_{k} \omega^{\sigma_{k}}, f_{k}=\prod_{r=1}^{n} \lambda_{k} y_{r, k}^{\mathrm{B}} / \tilde{y}_{r, k}^{\mathrm{B}}$. The reconstructed formula for $\mathcal{C}(\lambda)$ is

$$
\begin{aligned}
\mathcal{C}(\lambda) \Psi_{\boldsymbol{\sigma}}= & \frac{\theta-\lambda^{2}}{\lambda} \sum_{k=1}^{n} \prod_{r \neq k} \frac{\lambda_{k}\left(\lambda-\lambda_{r}\right)\left(\omega \theta-\lambda \lambda_{r}\right)}{\lambda\left(\lambda_{k}-\lambda_{r}\right)\left(\omega \theta-\lambda_{k} \lambda_{r}\right)} \\
& \times \frac{\lambda_{k}}{\omega \theta-\lambda_{k}^{2}}\left(\frac{\omega \theta-\lambda \lambda_{k}}{\theta-\lambda_{k}^{2}} \mathcal{C}\left(\lambda_{k}\right) \Psi_{\boldsymbol{\sigma}}-\frac{\omega \lambda_{k}\left(\lambda-\lambda_{k}\right)}{\omega^{2} \theta-\lambda_{k}^{2}} \mathcal{C}\left(\omega \theta / \lambda_{k}\right) \Psi_{\boldsymbol{\sigma}}\right) \\
& +\frac{1}{2} \sum_{\epsilon= \pm 1} \prod_{r=1}^{n} \frac{\left(\lambda-\lambda_{r}\right)\left(\omega \theta-\lambda \lambda_{r}\right)}{\lambda\left(\epsilon \sqrt{\theta}-\lambda_{r}\right)\left(\omega \epsilon \sqrt{\theta}-\lambda_{r}\right)}(1+\epsilon \lambda / \sqrt{\theta}) \mathcal{C}(\epsilon \sqrt{\theta}) \Psi_{\boldsymbol{\sigma}}
\end{aligned}
$$

Here we give some heuristic explanation of formulas (4.8). Let $\Psi_{\boldsymbol{\sigma}}$ be an eigenvector function of $\mathcal{A}(\lambda)$ satisfying (4.6). Then acting by both sides of commutation relation

$$
\begin{aligned}
(\omega \mu-\mu) & (\theta-\lambda \mu) \mathcal{C}(\lambda) \mathcal{A}(\mu)+(\mu-\lambda)(\omega \theta-\lambda \mu) \mathcal{A}(\lambda) \mathcal{C}(\mu) \\
& +(\mu-\lambda)(\omega \lambda \mu-\lambda \mu) \mathcal{B}(\lambda) \mathcal{A}(\mu)=(\theta-\lambda \mu)(\omega \mu-\lambda) \mathcal{C}(\mu) \mathcal{A}(\lambda),
\end{aligned}
$$

which follows from (2.4), on $\Psi_{\boldsymbol{\sigma}}$ at $\mu=\lambda_{k}$ we get

$$
\mathcal{A}(\lambda) \mathcal{C}\left(\lambda_{k}\right) \Psi_{\boldsymbol{\sigma}}=\frac{\left(\lambda-\omega \lambda_{k}\right)\left(\theta / \lambda-\lambda_{k}\right)}{\left(\lambda-\lambda_{k}\right)\left(\theta \omega / \lambda-\lambda_{k}\right)} \mathcal{C}\left(\lambda_{k}\right) \mathcal{A}(\lambda) \Psi_{\boldsymbol{\sigma}}
$$

Using (4.6) we see that $\mathcal{C}\left(\lambda_{k}\right) \Psi_{\boldsymbol{\sigma}}$ is an eigenvector of $\mathcal{A}(\lambda)$ with shifted zero, that is $\lambda_{k}$ replaced by $\omega \lambda_{k}$. It means that $\mathcal{C}\left(\lambda_{k}\right) \Psi_{\boldsymbol{\sigma}}$ is proportional to $\Psi_{\boldsymbol{\sigma}^{+k}}$. Clearly, this argumentation is not sufficient to prove the relations (4.8). The derivation of these relations can be carried out adapting the corresponding derivation from [5]. To prove (4.9) we use (4.1) at $\lambda= \pm \sqrt{\theta}$ and then (3.2). 


\section{Eigenvectors for the two-boundary RTC. Separation of variables}

From (2.6) it follows

$$
\mathcal{B}(\lambda)=\frac{\mathcal{C}(\lambda)(\omega-1) \theta+\mathcal{C}\left(\frac{\omega \theta}{\lambda}\right)\left(\theta-\lambda^{2}\right)}{\omega \theta-\lambda^{2}},
$$

which gives

$$
\begin{aligned}
\boldsymbol{t}(\lambda) & =\frac{\omega^{2} \theta-\lambda^{2}}{\lambda} \alpha_{+} \mathcal{A}(\lambda)+\eta_{+} \mathcal{C}(\lambda)+\eta_{+} \omega \mathcal{B}(\lambda) \\
& =\frac{\left(\omega^{2} \theta-\lambda^{2}\right)\left(\theta-\lambda^{2}\right)}{\lambda^{2}} \alpha_{+} \widetilde{\mathcal{A}}(\lambda)+\eta_{+} \frac{\mathcal{C}(\lambda)\left(\omega^{2} \theta-\lambda^{2}\right)+\omega \mathcal{C}\left(\frac{\omega \theta}{\lambda}\right)\left(\theta-\lambda^{2}\right)}{\omega \theta-\lambda^{2}} .
\end{aligned}
$$

We read off the structure of $\boldsymbol{t}(\lambda)$ from this expression

$$
\begin{aligned}
\boldsymbol{t}(\lambda)= & -\left(\omega^{2} \theta^{2} / \lambda^{2}-\theta\right)\left(\lambda^{2}-\theta\right) \frac{\alpha_{+} \alpha_{-} \varkappa^{n}}{\theta}\left((\lambda+\omega \theta / \lambda)^{n}+(\lambda+\omega \theta / \lambda)^{n-1} H_{1}^{\mathrm{BB}}+\cdots\right. \\
& \left.+(\lambda+\omega \theta / \lambda) H_{n-1}^{\mathrm{BB}}+H_{n}^{\mathrm{BB}}\right)+\eta_{+} \eta_{-} p_{n}(\lambda),
\end{aligned}
$$

where

$$
p_{n}(\lambda)=(\lambda+\omega \theta / \lambda) \theta^{(n-1) / 2} \quad \text { if } n \text { is odd, } \quad p_{n}(\lambda)=(1+\omega) \theta^{n / 2} \quad \text { if } n \text { is even }
$$

and the set of operators $H_{1}^{\mathrm{BB}}, H_{2}^{\mathrm{BB}}, \ldots, H_{n}^{\mathrm{BB}}$ should be identified with the commuting set of Hamiltonians for the two-boundary RTC. Explicitly,

$$
\begin{aligned}
H_{1}^{\mathrm{BB}}= & \varkappa \sum_{k=1}^{n}\left(\boldsymbol{v}_{k}+\omega \theta \varkappa^{-2} \boldsymbol{v}_{k}^{-1}\right)-\sum_{k=1}^{n-1}\left(\boldsymbol{u}_{k} \boldsymbol{u}_{k+1}^{-1} \boldsymbol{v}_{k+1}+\omega \theta \varkappa^{-2} \boldsymbol{u}_{k} \boldsymbol{u}_{k+1}^{-1} \boldsymbol{v}_{k}^{-1}\right) \\
& -\left(\eta_{-} / \alpha_{-}\right) \boldsymbol{u}_{1}^{-1} \boldsymbol{v}_{1}-\left(\eta_{+} / \alpha_{+}\right) \varkappa^{-1} \boldsymbol{v}_{n}^{-1} \boldsymbol{u}_{n} .
\end{aligned}
$$

Let $\Phi_{\mathbf{E}}, \mathbf{E}=\left(E_{1}, \ldots, E_{n}\right)$, be a common eigenvector for the commuting set of operators $\left\{H_{k}^{\mathrm{BB}}\right\}$ :

$$
H_{k}^{\mathrm{BB}} \Phi_{\mathbf{E}}=E_{k} \Phi_{\mathbf{E}}
$$

and $t_{\mathbf{E}}(\lambda)$ is corresponding eigenvalue polynomial for $\boldsymbol{t}(\lambda)$. In accordance with a procedure of the Separation of Variables we are looking for $\Phi_{\mathbf{E}}$ to be in the form

$$
\Phi_{\mathbf{E}}=\sum_{\boldsymbol{\sigma}} \prod_{i<j}\left(\left(\lambda_{i}-\lambda_{j}\right)\left(\omega \theta-\lambda_{i} \lambda_{j}\right)\right) \prod_{k=1}^{n} q_{k}\left(\sigma_{k}, \mathbf{E}\right) \Psi_{\boldsymbol{\sigma}},
$$

where $\lambda_{k}=-\varkappa s_{k} \omega^{\sigma_{k}}$.

Using the action formula (5.1) for $\boldsymbol{t}(\lambda)$ on $\Psi_{\boldsymbol{\sigma}}$ together with (4.6) and (4.10) we get Baxter equations for separated variables $q_{k}\left(\sigma_{k}, \mathbf{E}\right)$ :

$$
\begin{aligned}
t_{\mathbf{E}}\left(\lambda_{k}\right) q_{k}\left(\sigma_{k}, \mathbf{E}\right)= & \eta_{+} \eta_{-} \frac{\left(\theta-\lambda_{k}^{2}\right)}{\left(\omega \theta-\omega^{-2} \lambda_{k}^{2}\right)} \omega^{1-2 n} f_{k} q_{k}\left(\sigma_{k}-1, \mathbf{E}\right) \\
& +\eta_{+} \eta_{-} \frac{\left(\omega^{2} \theta-\lambda_{k}^{2}\right)}{\left(\theta-\omega \lambda_{k}^{2}\right)} \omega^{n-1} \theta^{n} f_{k}^{-1} q_{k}\left(\sigma_{k}+1, \mathbf{E}\right),
\end{aligned}
$$


where $f_{k}=\prod_{r=1}^{n} \lambda_{k} y_{r, k}^{\mathrm{B}} / \tilde{y}_{r, k}^{\mathrm{B}}$. The corresponding Baxter equation can be considered (for each $k=1,2, \ldots, n)$ as a homogeneous system of linear equations for $N$ unknowns $q_{k}(\sigma, \mathbf{E}), \sigma \in \mathbb{Z}_{N}$. The requirement of the existence of non-trivial solutions for all $k=1,2, \ldots, n$ fixes the common spectrum for the commuting set of operators $\left\{H_{k}^{\mathrm{BB}}\right\}$. Unfortunately, this problem can not be solved explicitly for general $N$. But if we know the spectrum we can construct the eigenvectors solving the systems of linear equations. We would like to report about such a progress [7] in the case of the periodic Baxter-Bazhanov-Stroganov model at $N=2$ which contains in particular the Ising model.

\section{Another proof of commutativity of $\boldsymbol{H}_{k}^{\mathrm{B}}$}

In this section we describe an alternative approach to the proof of commutativity of Hamiltonians using as an example the set of the one-boundary RTC Hamiltonians $H_{k}^{\mathrm{B}}$. This approach is based on the results from [12]. Let us consider the generation function

$$
\begin{aligned}
\mathcal{A}(\lambda) & =\alpha \prod_{k=1}^{n} \frac{\left(\lambda+\tilde{\boldsymbol{v}}_{k}\right)\left(\theta / \lambda+\tilde{\boldsymbol{v}}_{k}\right)}{\tilde{\boldsymbol{v}}_{k}}+\eta \sum_{k=1}^{n} \tilde{\boldsymbol{u}}_{r}^{-1} \tilde{\boldsymbol{v}}_{r} \prod_{s \neq k} \frac{\left(\lambda+\tilde{\boldsymbol{v}}_{s}\right)\left(\theta / \lambda+\tilde{\boldsymbol{v}}_{s}\right)}{\tilde{\boldsymbol{v}}_{r}-\tilde{\boldsymbol{v}}_{s}} \\
& =\alpha \prod_{k=1}^{n}\left(\Lambda+\tilde{\boldsymbol{v}}_{k}+\theta / \tilde{\boldsymbol{v}}_{k}\right)+\eta \sum_{k=1}^{n} \tilde{\boldsymbol{u}}_{k}^{-1} \tilde{\mathbf{V}} \prod_{s \neq k} \frac{\left(\Lambda+\tilde{\boldsymbol{v}}_{s}+\theta / \tilde{\boldsymbol{v}}_{s}\right)}{\tilde{\boldsymbol{v}}_{k}-\tilde{\boldsymbol{v}}_{s}}
\end{aligned}
$$

where $\Lambda=\lambda+\theta / \lambda, \tilde{\mathbf{V}}=\tilde{\boldsymbol{v}}_{1} \tilde{\boldsymbol{v}}_{2} \cdots \tilde{\boldsymbol{v}}_{n}$. The set of operators $\tilde{\boldsymbol{u}}_{j}, \tilde{\boldsymbol{v}}_{j}$ satisfy the relations

$$
\tilde{\boldsymbol{u}}_{j} \tilde{\boldsymbol{u}}_{k}=\tilde{\boldsymbol{u}}_{k} \tilde{\boldsymbol{u}}_{j}, \quad \tilde{\boldsymbol{v}}_{j} \tilde{\boldsymbol{v}}_{k}=\tilde{\boldsymbol{v}}_{k} \tilde{\boldsymbol{v}}_{j}, \quad \tilde{\boldsymbol{u}}_{j} \tilde{\boldsymbol{v}}_{k}=q^{\delta_{j, k}} \tilde{\boldsymbol{v}}_{k} \tilde{\boldsymbol{u}}_{j}
$$

where $q$ is a non-zero complex number. Note that this expression for $\mathcal{A}(\lambda)$ coincides with (4.2) (see also (A.3)) up to a redefinition of parameters.

Expanding $\mathcal{A}$ in $\Lambda$ we obtain

$$
\mathcal{A}=\alpha\left(\Lambda^{n}+H_{1}^{\mathrm{B}} \Lambda^{n-1}+\cdots+H_{n-1}^{\mathrm{B}} \Lambda+H_{n}^{\mathrm{B}}\right) .
$$

We will prove that the operators $H_{k}^{\mathrm{B}}$ obtained by such expansion are pairwise commuting. Let us introduce new operators $\Lambda_{k}=-\tilde{\boldsymbol{v}}_{k}-\theta / \tilde{\boldsymbol{v}}_{k}$. Taking into account

$$
\Lambda_{k}-\Lambda_{s}=\left(\tilde{\boldsymbol{v}}_{k}-\tilde{\boldsymbol{v}}_{s}\right)\left(\theta /\left(\tilde{\boldsymbol{v}}_{k} \tilde{\boldsymbol{v}}_{s}\right)-1\right),
$$

we get

$$
\mathcal{A}=\alpha \prod_{k=1}^{n}\left(\Lambda-\Lambda_{k}\right)+\eta \sum_{k=1}^{n} \tilde{Z}_{k} \prod_{s \neq k} \frac{\Lambda-\Lambda_{s}}{\Lambda_{k}-\Lambda_{s}},
$$

where

$$
\tilde{Z}_{k}=\tilde{\boldsymbol{u}}_{k}^{-1} \tilde{\boldsymbol{v}}_{k}^{n} \prod_{s \neq k} \frac{\tilde{\boldsymbol{v}}_{s}^{2}}{\left(\theta-\tilde{\boldsymbol{v}}_{k} \tilde{\boldsymbol{v}}_{s}\right)} .
$$

Finally using the interpolation formula for the polynomial $\prod_{k=1}^{n}\left(\Lambda-\Lambda_{k}\right)-\Lambda^{n}$ of degree $n-1$ :

$$
\prod_{k=1}^{n}\left(\Lambda-\Lambda_{k}\right)=\Lambda^{n}-\sum_{k=1}^{n} \Lambda_{k}^{n} \prod_{s \neq k} \frac{\Lambda-\Lambda_{s}}{\Lambda_{k}-\Lambda_{s}},
$$


we have

$$
\mathcal{A}=\alpha \Lambda^{n}+\alpha \sum_{k=1}^{n} Z_{k} \prod_{s \neq k} \frac{\Lambda-\Lambda_{s}}{\Lambda_{k}-\Lambda_{s}}
$$

where $Z_{k}=\eta \tilde{Z}_{k} / \alpha-\Lambda_{k}^{n}$. It is easy to verify that

$$
\left[\Lambda_{j}, \Lambda_{k}\right]=0, \quad\left[Z_{j}, Z_{k}\right]=0, \quad\left[Z_{j}, \Lambda_{k}\right]=0, \quad j \neq k .
$$

Theorem 1. For the set of operators $\Lambda_{k}, Z_{k}, k=1,2, \ldots, n$, satisfying (6.4), we have commutativity of operators $H_{k}^{\mathrm{B}}$ def ined by expansion (6.2) of $\mathcal{A}$ given by (6.3).

Proof. Let $\boldsymbol{f}$ be the $n$ by $n+1$ matrix with the matrix elements $f_{i, j}, 1 \leq i \leq n, 0 \leq j \leq n$, such that any two of them belonging to different rows are commuting. Denote by $\Delta_{j}, 0 \leq j \leq n$, the determinant of matrix $\boldsymbol{f}$ with $j$-th column omitted. Then from [12] it follows that the operators $H_{k}=\Delta_{k} \Delta_{0}^{-1}, k=1,2, \ldots, n$, are pairwise commuting.

Let us fix the matrix elements of $\boldsymbol{f}$ by the matrix elements $f_{i, 0}=Z_{i}, f_{i, k}=\Lambda_{i}^{n-k}$, where $i$, $k=1,2, \ldots, n$. In this case we have $\Delta_{0}=\prod_{m<l}\left(\Lambda_{m}-\Lambda_{l}\right)$. Expanding $\Delta_{s}, s \geq 1$, with respect to first column we have

$$
\Delta_{s}=\sum_{k=1}^{n} Z_{k}(-1)^{k+1} \Delta_{k ; 0, s}
$$

where $\Delta_{k ; 0, s}$ is the determinant of matrix $\boldsymbol{f}$ with $k$-th row and $0, s$-th columns omitted. Therefore for the generating function for commuting $H_{s}=\Delta_{s} \Delta_{0}^{-1}$ we get

$$
\sum_{s=1}^{n}(-1)^{s+1} H_{s} \Lambda^{n-s}=\sum_{k=1}^{n} Z_{k}\left(\sum_{s=1}^{n}(-1)^{k+s} \Lambda^{n-s} \Delta_{k ; 0, s}\right) \Delta_{0}^{-1} .
$$

The expression in the brackets is the determinant expanded with respect to $k$-th row of $\boldsymbol{f}$ with 0 -th column omitted and all $\Lambda_{k}$ replaced by $\Lambda$. Hence this expression is similar to $\Delta_{0}$ but with $\Lambda_{k}$ replaced by $\Lambda$. It gives

$$
\sum_{s=1}^{n}(-1)^{s+1} H_{s} \Lambda^{n-s}=\sum_{k=1}^{n} Z_{k} \prod_{s \neq k} \frac{\Lambda-\Lambda_{s}}{\Lambda_{k}-\Lambda_{s}} .
$$

Comparing this formula with (6.2) and (6.3) we get $H_{k}^{\mathrm{B}}=(-1)^{k+1} H_{k}$ and therefore the commutativity of $H_{k}^{\mathrm{B}}$.

Taking into account the discussion before the theorem we get that the function (6.1) is a generating function for the commuting set of operators $H_{k}^{\mathrm{B}}$.

\section{Discussion}

We had applied in this contribution the Separation of Variables to obtain the commuting Hamiltonians eigenvectors of the quantum relativistic Toda chain at a root of unity with boundary interaction. As we already have discussed in Introduction, the $L$-operator of the relativistic quantum Toda chain at a root of unity is a special case of the cyclic $L$-operator of the BaxterBazhanov-Stroganov model (BBS). It was shown in the papers $([7,20])$, that the BBS model at $N=2$ coincides with the free fermion point of the generalized Ising model. We plan to apply the Separation of Variables method, used in this contribution, to the $N=2 \mathrm{BBS}$ model with integrable boundary conditions. 


\section{A Derivation of the action formula for $\widetilde{\mathcal{A}}(\lambda)$}

In this Appendix we will prove the formula

$$
\widetilde{\mathcal{A}}(\lambda) \Psi_{\boldsymbol{\sigma}}=\alpha_{-}(-\varkappa)^{n} \prod_{k=1}^{n}\left(\frac{\left(\lambda-\lambda_{k}\right)\left(\omega \theta / \lambda-\lambda_{k}\right)}{\lambda_{k}}\right) \Psi_{\boldsymbol{\sigma}},
$$

where the eigenvector $\Psi_{\boldsymbol{\sigma}}$ is defined by (4.4), (4.5). In what follows we use the short notations $\lambda_{n, k}=-\varkappa v_{n, k} \omega^{\gamma_{n, k}}$ and $\lambda_{k}=-\varkappa s_{k} \omega^{\sigma_{k}}$.

To prove (A.1) we use the expression (4.2)

$$
\widetilde{\mathcal{A}}(\lambda)=(\omega \theta)^{n} \mathbf{V}^{-1}\left[\alpha_{-} A(\omega \theta / \lambda) A(\lambda)+\frac{\eta_{-} \lambda}{\left(\theta-\lambda^{2}\right)}(A(\omega \theta / \lambda) C(\lambda)-C(\omega \theta / \lambda) A(\lambda))\right],
$$

and the action formulas

$$
\begin{aligned}
& A(\lambda)\left|\gamma_{n}\right\rangle=\prod_{k=1}^{n}\left(1-\frac{\lambda_{n, k}}{\lambda}\right)\left|\gamma_{n}\right\rangle, \quad C(\lambda)\left|\gamma_{n}\right\rangle=\sum_{k=1}^{n} \prod_{r \neq k}\left(\frac{\lambda_{n, k}\left(\lambda-\lambda_{n, r}\right)}{\lambda\left(\lambda_{n, k}-\lambda_{n, r}\right)}\right) C\left(\lambda_{n, k}\right)\left|\gamma_{n}\right\rangle, \\
& C\left(\lambda_{n, k}\right)\left|\gamma_{n}\right\rangle=\frac{\prod_{m=1}^{n} \lambda_{n, m}}{(-\varkappa)^{n} h_{k}\left(\omega \lambda_{n, k}\right)^{n-1}}\left|\gamma_{n}^{+k}\right\rangle .
\end{aligned}
$$

We have

$$
\begin{aligned}
& (A(\omega \theta / \lambda) C(\lambda)-C(\omega \theta / \lambda) A(\lambda))\left|\gamma_{n}\right\rangle \\
& \quad=\frac{\theta-\lambda^{2}}{\theta \lambda} \sum_{k=1}^{n} \lambda_{n, k}^{n} \prod_{r \neq k}\left[\left(1-\frac{\lambda \lambda_{n, r}}{\omega \theta}\right)\left(1-\frac{\lambda_{n, r}}{\lambda}\right) \frac{1}{\lambda_{n, k}-\lambda_{n, r}}\right] C\left(\lambda_{n, k}\right)\left|\gamma_{n}\right\rangle .
\end{aligned}
$$

Therefore

$$
\begin{aligned}
\widetilde{\mathcal{A}}(\lambda)\left|\gamma_{n}\right\rangle= & (\omega \theta)^{n}\left\{\alpha_{-}(-\varkappa)^{n} \prod_{k=1}^{n} \frac{\left(1-\lambda \lambda_{n, k} /(\omega \theta)\right)\left(1-\lambda_{n, k} / \lambda\right)}{\lambda_{n, k}}\left|\gamma_{n}\right\rangle\right. \\
& \left.+\frac{\eta_{-}}{\theta} \sum_{k=1}^{n} \lambda_{n, k}^{n} \prod_{r \neq k}\left[\left(1-\frac{\lambda \lambda_{n, r}}{\omega \theta}\right)\left(1-\frac{\lambda_{n, r}}{\lambda}\right) \frac{1}{\lambda_{n, k}-\lambda_{n, r}}\right] \frac{\omega^{-1}}{h_{k}\left(\omega \lambda_{n, k}\right)^{n-1}}\left|\gamma_{n}^{+k}\right\rangle\right\} .
\end{aligned}
$$

Acting $\widetilde{\mathcal{A}}(\lambda)$ on $\Psi_{\boldsymbol{\sigma}}$ and shifting in an appropriate way the summation we get

$$
\begin{aligned}
\widetilde{\mathcal{A}}(\lambda) \Psi_{\boldsymbol{\sigma}} & =\sum_{\gamma_{n}} Q\left(\boldsymbol{\gamma}_{n}, \boldsymbol{\sigma}\right) \widetilde{\mathcal{A}}(\lambda)\left|\gamma_{n}\right\rangle \\
& =(\omega \theta)^{n} \sum_{\gamma_{n}} Q\left(\boldsymbol{\gamma}_{n}, \boldsymbol{\sigma}\right)\left\{\alpha_{-}(-\varkappa)^{n} \prod_{k=1}^{n} \frac{\left(1-\lambda \lambda_{n, k} /(\omega \theta)\right)\left(1-\lambda_{n, k} / \lambda\right)}{\lambda_{n, k}}\right. \\
& \left.+\frac{\eta_{-}}{\theta} \sum_{k=1}^{n} \prod_{r \neq k}\left[\left(1-\frac{\lambda \lambda_{n, r}}{\omega \theta}\right)\left(1-\frac{\lambda_{n, r}}{\lambda}\right) \frac{1}{\omega^{-1} \lambda_{n, k}-\lambda_{n, r}}\right] \frac{\lambda_{n, k}}{h_{k} \omega^{n+1}} \frac{Q\left(\gamma_{n}^{-k}, \boldsymbol{\sigma}\right)}{Q\left(\boldsymbol{\gamma}_{n}, \boldsymbol{\sigma}\right)}\right\}\left|\boldsymbol{\gamma}_{n}\right\rangle .
\end{aligned}
$$

Using (4.5) and (3.1) and then (4.7) we obtain

$$
\frac{Q\left(\boldsymbol{\gamma}_{n}^{-k}, \boldsymbol{\sigma}\right)}{Q\left(\boldsymbol{\gamma}_{n}, \boldsymbol{\sigma}\right)}=\frac{\varkappa^{2} v_{n, k}^{2}}{\lambda_{n, k}^{2}} \prod_{m=1}^{n} \frac{\left(\lambda_{m}-\lambda_{n, k}\right)\left(1-\lambda_{m} \lambda_{n, k} /(\omega \theta)\right)}{\lambda_{m} y_{k, m}^{\mathrm{B}} \tilde{y}_{k, m}^{\mathrm{B}}}
$$




$$
\begin{aligned}
& \times \prod_{r \neq k}\left(\frac{\omega^{-1} \lambda_{n, k}-\lambda_{n, r}}{\lambda_{n, k}-\lambda_{n, r}} \frac{\tilde{y}_{k, r}}{\left(1-\lambda_{n, k} \lambda_{n, r} /(\omega \theta)\right)}\right) \\
= & \frac{\alpha_{-} \theta \varkappa^{n} \omega^{n+1} h_{k}}{\eta_{-} \lambda_{n, k}^{2}} \prod_{m=1}^{n} \frac{\left(\lambda_{m}-\lambda_{n, k}\right)\left(1-\lambda_{m} \lambda_{n, k} /(\omega \theta)\right)}{\lambda_{m}} \\
& \times \prod_{r \neq k}\left(\frac{\omega^{-1} \lambda_{n, k}-\lambda_{n, r}}{\lambda_{n, k}-\lambda_{n, r}} \frac{1}{\left(1-\lambda_{n, k} \lambda_{n, r} /(\omega \theta)\right)}\right) .
\end{aligned}
$$

Finally, taking into account the identity

$$
\prod_{k=1}^{n}\left(\Lambda-\Lambda_{n, k}\right)+\sum_{m=1}^{n} \prod_{r \neq m} \frac{\Lambda-\Lambda_{n, r}}{\Lambda_{n, m}-\Lambda_{n, r}} \prod_{k=1}^{n}\left(\Lambda_{n, m}-\Lambda_{k}\right)=\prod_{k=1}^{n}\left(\Lambda-\Lambda_{k}\right)
$$

with

$$
\Lambda=-\lambda-\omega \theta / \lambda, \quad \Lambda_{k}=-\lambda_{k}-\omega \theta / \lambda_{k}, \quad \Lambda_{n, k}=-\lambda_{n, k}-\omega \theta / \lambda_{n, k}
$$

we get (A.1).

\section{Acknowledgements}

The research presented here is supported by the French-Ukrainian project "Dnipro" and the Ukrainian State Foundation for Fundamental Research. The work of V.S. was also partially supported by the SCOPES-project IB7320-110848 of Swiss NSF. V.R. warmly acknowledged a partial support of grants RFBR 06-02-17382 and NSh-8065.2006.2 as well as the ANR-2005 ("Geometry and Integrability in Mathematical Physics") support.

\section{References}

[1] Sklyanin E., Separation of variable. New trends, Prog. Theoret. Phys. Suppl. 118 (1995), 35-60, solv-int/9504001.

[2] Kharchev S., Lebedev D., Integral representation for the eigenfunctions of quantum periodic Toda chain, Lett. Math. Phys. 50 (1999), 53-77, hep-th/9910265.

[3] Kharchev S., Lebedev D., Semenov-Tian-Shansky M., Unitary representations of $U_{q}(s l(2, R))$, the modular double, and the multiparticle $q$-deformed Toda chains, Comm. Math. Phys. 225 (2002), 573-609, hep-th/0102180.

[4] Kuznetsov V., Separation of variables for the $D_{n}$ type periodic Toda lattice, J. Phys. A: Math. Gen. 30 (1997), 2127-2138, solv-int/9701009.

[5] Iorgov N., Shadura V., Wave functions of the Toda chain with boundary interactions, Theor. Math. Phys. 142 (2005), 289-305, nlin.SI/0411002.

[6] Sklyanin E., Boundary conditions for integrable quantum systems, J. Phys. A: Math. Gen. 21 (1988), $2375-2389$.

[7] von Gehlen G., Iorgov N., Pakuliak S., Shadura V., Baxter-Bazhanov-Stoganov model: separation of variables and Baxter equation, J. Phys. A: Math. Gen. 39 (2006), 7257-7282, nlin.SI/0603028.

[8] Iorgov N., Eigenvectors of open Bazhanov-Stroganov quantum chain, SIGMA 2 (2006), 019, 10 pages, nlin.SI/0602010.

[9] Bazhanov V.V., Stroganov Yu.G., Chiral Potts model as a descendant of the six-vertex model, J. Statist. Phys. 59 (1990), 799-817.

[10] Korepanov I.G., Hidden symmetries in the 6-vertex model of statistical physics, Zap. Nauchn. Sem. S.-Peterburg. Otdel. Mat. Inst. Steklov. (POMI) 215 (1994), 163-177 (English transl.: J. Math. Sci. (New York) 85 (1997), 1661-1670, hep-th/9410066). 
[11] Pakuliak S., Sergeev S., Quantum relativistic Toda chain at root of unity: isospectrality, modified $Q$-operator and functional Bethe ansatz, Int. J. Math. Math. Sci. 31 (2002), 513-554, nlin.SI/0205037.

[12] Enriquez B., Rubtsov V., Commuting families in skew fields and quantization of Beauville's fibrations, Duke Math. J. 82 (2003), 197-219, math.AG/0112276.

[13] Sergeev S., Coefficient matrices of a quantum discrete auxiliary linear problem, Zap. Nauchn. Sem. POMI 269 (2000), no. 16, 292-307 (in Russian).

[14] Babelon O., Talon M., Riemann surfaces, separation of variables and classical and quantum integrability, Phys. Lett. A 312 (2003), 71-77, hep-th/0209071.

[15] Kuznetsov V.B., Tsiganov A.V., Infinite series of Lie algebras and boundary conditions for integrable systems, J. Sov. Math. 59 (1992), 1085-1092.

[16] Kuznetsov V.B., Tsiganov A.V., Separation of variables for the quantum relativistic Toda lattices, Report 94-07, Mathematical Preprint Series, University of Amsterdam, 1994, hep-th/9402111.

[17] Kuznetsov V.B., Jorgensen M.F., Christiansen P.L., New boundary conditions for integrable lattices, J. Phys. A: Math. Gen. 28 (1995), 4639-4654, hep-th/9503168.

[18] de Vega H.J., Gonzalez-Ruiz A., Boundary K-matrices for the XYZ, XXZ and XXX spin chains, J. Phys. A: Math. Gen. 28 (1994), 6129-6141.

[19] Bazhanov V.V., Baxter R.J., Star-triangle relation for a three dimensional model, J. Statist. Phys. 71 (1993), 839-864, hep-th/9212050.

[20] Bugrij A.I., Iorgov N.Z., Shadura V.N., Alternative method of calculating the eigenvalues of the transfer matrix of the $\tau_{2}$ model for $N=2$, JETP Lett. 119 (2005), no. 2, 311-315. 